\title{
Singularity confinement and chaos in discrete systems
}

\author{
Jarmo Hietarinta $^{1}$ and Claude Viallet ${ }^{2}$ \\ ${ }^{1}$ Department of Physics, University of Turku, FIN-20014 Turku, Finland \\ ${ }^{2}$ CNRS and Université Paris VI, Boîte 126, 4 place Jussieu, F-75252 Paris Cedex 05, France
}

We present a number of second order maps, which pass the singularity confinement test commonly used to identify integrable discrete systems, but which nevertheless are nonintegrable. As a more sensitive integrability test, we propose the analysis of the complexity ("algebraic entropy") of the map using the growth of the degree of its iterates: integrability is associated with polynomial growth while the generic growth is exponential for chaotic systems.

05.50.+q,02.90.+p

Discrete systems have for a long time been a subject of study in the field of dynamical systems. A strong practical motivation is the power of numerical exploration for such systems [1], which led to interesting findings in chaos theory. On the other hand, several numerical algorithms ("convergence acceleration algorithms") are related to integrable discrete maps (see [2] and references therein). Finding out whether a given system is chaotic or integrable is then a basic question worth more investigation. One open problem to find an algorithmic test of integrability, and this is the subject of the present letter.

In the case of continuous systems one can test for the "Painlevé property" [3 6], which is closely related to integrability and has considerable predictive power. An analogue of the Painlevé test for discrete systems was proposed in [7] and has been used as a powerful constructive tool, e.g., to identify discrete Painlevé equations [8]. This singularity confinement test is similar in spirit to the continuous Painlevé test in that it analyses behavior around a movable singularity of the map. When a map is iterated, it may happen that we reach a point for which the next value is ill-defined due to the appearance of an indeterminate form, $\infty-\infty, 0 \cdot \infty$ or such. One should then study the behavior around the singularity: if the map can be continued in a way which allows, after a finite number of steps, to exit from the singularity without loss of information, then the system is said to pass the test.

We show in this letter that the confinement test is not sufficient to ensure integrability. We also propose another indicator for rational maps: a measure of the "algebraic entropy" 9.10], which has to do with global properties of the system (see later).

We shall consider the map

$$
x_{n+1}+x_{n-1}=x_{n}+a / x_{n}^{2},
$$

and some of its generalizations. Relation (11) defines a map $\left(x_{n-1}, x_{n}\right) \rightarrow x_{n+1}$. The potential singularity of this map is reached if, at some step (say step 0), we arrive at $x_{0}=0$ (with a finite nonzero $x_{-1}$ ), because then $x_{1}=\infty$, $x_{2}=\infty$, but $x_{3}=\infty-\infty$ and it is not clear how to proceed. To refine the analysis, let us assume that we arrive at $x_{0}=\epsilon$ by $x_{-1}=u$, with suitable previous $x_{n}$ 's. With these initial values we get the sequence (here $a=1$ )

$$
\begin{aligned}
x_{-1} & =u, \\
x_{0} & =\epsilon, \\
x_{1} & =\epsilon^{-2}-u+\epsilon, \\
x_{2} & =\epsilon^{-2}-u+\epsilon^{4}+\ldots, \\
x_{3} & =-\epsilon+2 \epsilon^{4}+\ldots, \\
x_{4} & =u-\epsilon+\ldots
\end{aligned}
$$

In this case the outcome is: " $\infty-\infty=0$ " and the sequence emerges from the singularity with the value $u$, i.e., without losing the initial information. This means that the system (11) passes the singularity confinement test without problems; the singularity structure $u \rightarrow \epsilon \rightarrow$ $\epsilon^{-2} \rightarrow \epsilon^{-2} \rightarrow-\epsilon \rightarrow u$ is rather typical.

The problem is that system (11) is chaotic, as we shall show. Our suspicion about the non-integrability of (1) arose when, motivated by [10], we evaluated the growth of the degree of its iterates as follows:

We start by writing the map as a first order twodimensional map

$$
\varphi: p_{n}=\left(x_{n-1}, x_{n}\right) \longrightarrow p_{n+1}=\left(x_{n}, x_{n+1}\right),
$$

and then rewrite $\varphi$ in terms of homogeneous coordinates $\left[y_{n}, z_{n}, t_{n}\right]$ by setting

$$
p_{n}=\left(\frac{z_{n}}{t_{n}}, \frac{y_{n}}{t_{n}}\right) .
$$

This means that we are now working in the twodimensional projective space $C P_{2}$, and that points with homogeneous coordinates $[y, z, t]$ and $[\lambda y, \lambda z, \lambda t]$ are to be identified (projectivization). For (1) the map $\varphi$ may be written as

$$
\varphi:\left[\begin{array}{c}
y \\
z \\
t
\end{array}\right] \rightarrow\left[\begin{array}{c}
y^{3}+a t^{3}-y^{2} z \\
y^{3} \\
t y^{2}
\end{array}\right] .
$$

In $\mathrm{CP}_{2}$ the above singularity pattern looks as follows:

$$
\left[\begin{array}{l}
0 \\
u \\
1
\end{array}\right] \rightarrow\left[\begin{array}{l}
1 \\
0 \\
0
\end{array}\right] \rightarrow\left[\begin{array}{l}
1 \\
1 \\
0
\end{array}\right] \rightarrow\left[\begin{array}{l}
0 \\
1 \\
0
\end{array}\right] \rightarrow\left[\begin{array}{l}
0 \\
0 \\
0
\end{array}\right]
$$


The last term of this sequence is not in $C P_{2}$ and is now the manifestation of the ambiguity mentioned above. Note also that in this formulation it is clear that infinities are not singularities: they look like any other point, the last component is just zero. The expansion around the singularity clarifies the situation. We get the sequence

$$
\begin{aligned}
{\left[\begin{array}{c}
\epsilon \\
u \\
1
\end{array}\right] \rightarrow\left[\begin{array}{r}
1-u \epsilon^{2}+\ldots \\
\epsilon^{3} \\
\epsilon^{2}
\end{array}\right] \rightarrow\left[\begin{array}{r}
1-3 u \epsilon^{2}+\ldots \\
1-3 u \epsilon^{2}+\ldots \\
\epsilon^{2}+\ldots
\end{array}\right] } \\
\rightarrow\left[\begin{array}{r}
-\epsilon^{3}+\ldots \\
1-9 u \epsilon^{2}+\ldots \\
\epsilon^{2}+\ldots
\end{array}\right] \\
\rightarrow\left[\begin{array}{r}
u \epsilon^{8}+\ldots \\
-\epsilon^{9}+\ldots \\
\epsilon^{8}+\ldots
\end{array}\right]=\left[\begin{array}{r}
u+\ldots \\
-\epsilon+\ldots \\
1+\ldots
\end{array}\right],
\end{aligned}
$$

and in the last term we are able to cancel the factor $\epsilon^{8}$, after which we can let $\epsilon \rightarrow 0$, getting $[u, 0,1]$. We have thus emerged from the singularity with the initial information $u$.

The cancellation mentioned here is crucial. It occurs only if there is a singularity in the map, because the existence of a singularity means that there will be common factors of $\epsilon$. Such cancellations of common factors are necessary to reduce the growth of the degree, because otherwise the successive iterates $\varphi^{(n)}$ of $\varphi$ would be polynomials of degree $d^{n}$, where $d$ is the degree of $\varphi$. For integrable systems the cancellations are in fact so strong that asymptotically the degree grows only polynomially.

The degree of $\varphi$ is not canonical, since it is not invariant under coordinate changes. However the growth of the degree is canonical [9]. It is generically exponential [11], but may become polynomial if the number of common factors is large enough. The conjecture is that integrability of the map implies polynomial growth [10 (see also 12]).

For the map (4) we get the following sequence of degrees: 1, 3, 9, 27, 73, 195, 513, 1347, 3529... The first four degrees follow the $3^{n}$ rule; cancellations then take place. Note that the first drop of the degree is $3 \times 27-73=8$ corresponding to the factorization of $\epsilon^{8}$ in the above calculation. From the nine first numbers of this sequence we inferred the generating function for the degrees:

$$
g(x)=\frac{1+3 x^{3}}{(1-x)(1+x)\left(x^{2}-3 x+1\right)} .
$$

The next degree, found by iteration of $\varphi$ is 9243 and coincides with the prediction obtained by expanding $g$ :

$$
\begin{aligned}
g= & 1+3 x+9 x^{2}+27 x^{3}+73 x^{4}+195 x^{5}+513 x^{6}+ \\
& +1347 x^{7}+3529 x^{8}+9243 x^{9}+24201 x^{10}+\ldots
\end{aligned}
$$

(A proof that (5) indeed is the generating function of the degrees will be given elsewhere [9].)
Function (5) generates a sequence with exponential growth. The denominator of $g$ contains two basic pieces of information. It first shows that the sequence of degrees verifies the very specific relation

$$
d_{n+4}-d \cdot d_{n+3}+d \cdot d_{n+1}-d_{n}=0,
$$

where $d=d_{1}=3$ is the degree of the map $\varphi$, and $d_{n}$ the actual degree of $\varphi^{n}$, after "projectivization". It also determines the asymptotic behavior of $d_{n}$ : if $\alpha$ is the smallest modulus of the roots of the denominator of (5), then $d_{n+1} \approx \alpha^{-1} d_{n}$ asymptotically. In this case $\alpha=$ $(3-\sqrt{5}) / 2$ and we define the "algebraic entropy" of the map by

$$
\mathcal{E} \equiv \lim _{n \rightarrow \infty} \frac{1}{n} \log \left(d_{n}\right)=\log \left(\frac{3+\sqrt{5}}{2}\right) .
$$

This calculation indicates that the map has nonvanishing entropy, and therefore is likely to be nonintegrable.

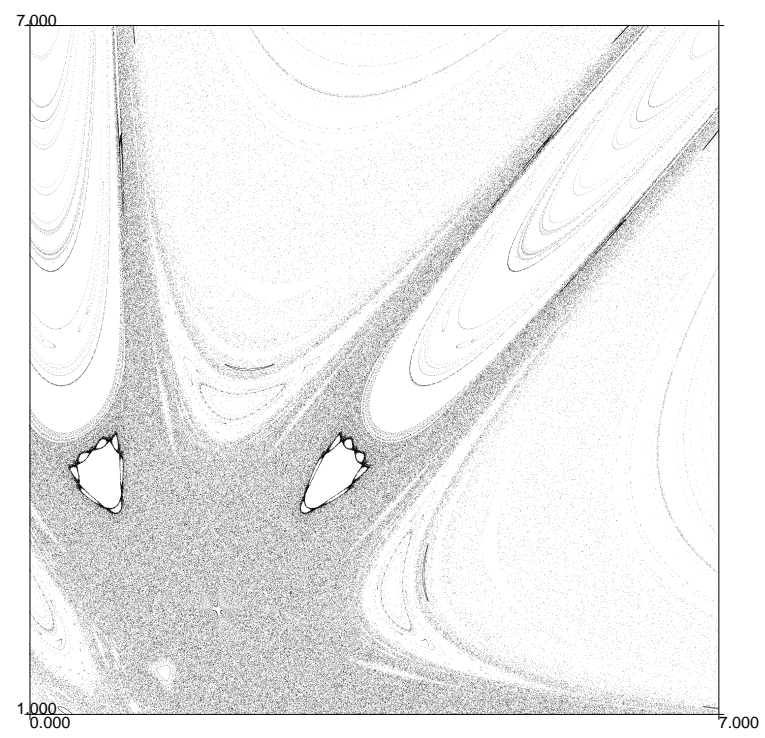

Fig. 1: A collection of orbits of the map (1)

Numerical chaos can be seen when we draw a picture of some orbits of the map, see Figure 1. This figure was obtained with $a=7$. The two "cat's paws" are around two points of a nine-periodic orbit of $\varphi$. Such a point of order nine of $\varphi\left(x_{n}=x_{n+1} \simeq 3.043896 \ldots\right)$ is located at the lower left hand corner of Figure 2. The picture is characteristic of chaotic behavior of a two dimensional conservative system.

In order to compare with a truly integrable system let us consider 14.

$$
x_{n+1}+x_{n-1}=\frac{a}{x_{n}^{2}}+\frac{b}{x_{n}},
$$

which is related to $d-P_{I}$. This is also a third degree map, but the singularity structure is now such that the degrees 
grow only as $1,3,9,19,33,51,73,99,129,163,201, \ldots$ The generating functional is

$$
g=\frac{1+3 x^{2}}{(1-x)^{3}}
$$

and in fact the degrees grow polynomially according to the simple rule

$$
d_{n}=2 n^{2}+1
$$

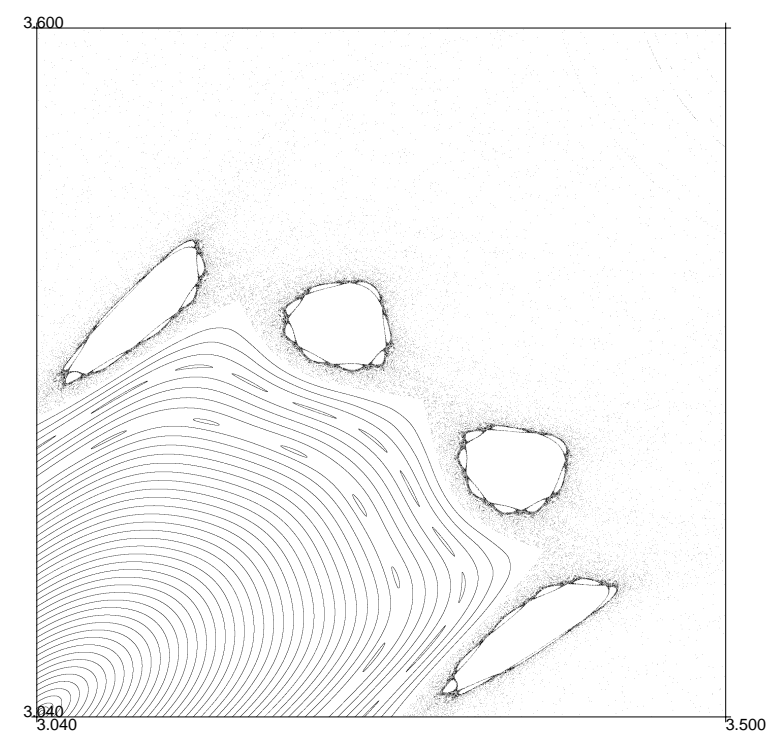

Fig. 2: A close up of Figure 1

If model (11) were an isolated example, it might be dismissed by some ad hoc rule. However, it turns out that the singularity confinement test is somewhat insensitive in general, and maps containing rather arbitrary functions pass the test. Let us consider the more general map

$$
x_{n+1}+x_{n-1}=x_{n}+f\left(x_{n}\right),
$$

where $f$ does not have to be rational. (Indeed, there is no inherent reason to limit the singularity confinement test to rational maps, while the notion of degree, and consequently the definition of the algebraic entropy is tightly related to (bi)-rationality. For non-rational transformations, one should use a definition of entropy more closely inspired from 111.)

It is clear that the map (10) can be iterated forwards and backwards except possibly when $f$ diverges. Let us therefore assume that $f$ diverges at some points $x_{j}^{*}$ with a power series expansion starting as

$$
f\left(x_{j}^{*}+\epsilon\right)=a_{j} \epsilon^{-K_{j}}(1+O(\epsilon)), K_{j}>0,
$$

and that it vanishes at infinity as

$$
f\left(\epsilon^{-1}\right)=b \epsilon^{L}(1+O(\epsilon)), L>0 .
$$

From these assumptions it follows in particular that $f\left(f\left(x_{j}^{*}+\epsilon\right)+O(1)\right)=b a_{j}^{-L} \epsilon^{L K_{j}}(1+O(\epsilon))$. The singularity analysis proceeds now as follows

$$
\begin{aligned}
x_{-1} & =u, \\
x_{0} & =x_{j}^{*}+\epsilon, \\
x_{1} & =f\left(x_{j}^{*}+\epsilon\right)-u+x_{j}^{*}+\epsilon, \\
x_{2} & =f\left(x_{j}^{*}+\epsilon\right)-u+b a_{j}^{-L} \epsilon^{L K_{j}}(1+O(\epsilon)), \\
x_{3} & =-x_{j}^{*}-\epsilon+2 b a_{j}^{-L} \epsilon^{L K_{j}}(1+O(\epsilon)), \\
x_{4} & =u-x_{j}^{*}+\Delta_{j}(\epsilon)+O(\epsilon),
\end{aligned}
$$

with

$\Delta_{j}(\epsilon)=f\left(-x_{j}^{*}-\epsilon+2 b a_{j}^{-L} \epsilon^{L K_{j}}(1+O(\epsilon))\right)-f\left(x_{j}^{*}+\epsilon\right)$.

Thus in order for $f$ to pass the singularity confinement test, we only need to impose the condition that

$$
\Delta_{j}(\epsilon)=O(1), \forall j
$$

and that this term does not cancel the $u$ dependence in $x_{4}$.

A simple calculation shows that (13) is true at least if 1) $K_{j}(L-1) \geq 1$ and 2) the singularity structure of $f$ is even, i.e., both $+x_{j}^{*}$ and $-x_{j}^{*}$ are singular points of $f$ and the expansions at these points match as $f\left(x_{j}^{*}+\epsilon\right)-f\left(-x_{j}^{*}-\epsilon\right)=O(\epsilon)$. The simplest such function is $x^{-2}$ (yielding the map (1)), but it is easy to construct other examples. If attention is restricted to rational functions, we can, e.g., pick any two relatively prime polynomials $Q(x)$ and $P(x)$ of degree $M$ and define $f(x)=(P(x) P(-x)) /\left(x^{2} Q(x) Q(-x)\right)$. As an example we performed the degree growth analysis above on the special case $P=x+5, Q=x+3$ and obtained entropy $\mathcal{E}=\log ((5+\sqrt{21}) / 2)$. Drawing the orbits again corroborates the claim of non-integrability.

Another class of maps which passes the singularity confinement test is contained in

$$
x_{n+1}+x_{n-1}=f_{n}\left(x_{n}\right),
$$

where we just assume that the functions $f_{n}$ diverge at some points $x_{j}^{*}$ (independent of $n$ ), and vanishes at infinity, but it is not necessary yet to specify how. Here we allow non-autonomous maps, i.e., $f$ may also depend on $n$, as indicated by the subscript (of course this could have been done with the previous model as well). The singularity analysis now goes as follows:

$$
\begin{aligned}
x_{-1} & =u, \\
x_{0} & =x_{j}^{*}+\epsilon, \\
x_{1} & =-u+f_{0}\left(x_{j}^{*}+\epsilon\right), \\
x_{2} & =-x_{j}^{*}-\epsilon+f_{1}\left(f_{0}\left(x_{j}^{*}+\epsilon\right)-u\right), \\
x_{3} & =u-f_{0}\left(x_{j}^{*}+\epsilon\right)+f_{2}\left(-x_{j}^{*}-\epsilon+f_{1}\left(f_{0}\left(x_{j}^{*}+\epsilon\right)-u\right)\right) .
\end{aligned}
$$


For the singularity to be confined at this step, it is sufficient that the behaviors at the singular points and at infinity match so that

$$
\begin{aligned}
\forall j, n \quad \lim _{\epsilon \rightarrow 0}\left[f_{n}\left(x_{j}^{*}+\epsilon\right)\right. & \\
& \left.-f_{n+2}\left(-x_{j}^{*}-\epsilon+f_{n+1}\left(f_{n}\left(x_{j}^{*}+\epsilon\right)-u\right)\right)\right]=0,
\end{aligned}
$$

The singularity pattern is shorter than for (11), $u \rightarrow 0 \rightarrow$ $\infty \rightarrow 0 \rightarrow \infty-\infty$, and upon expanding the ambiguity resolves to $u$. The confinement condition (15) is similar to (13).

The above results show that the singularity confinement test is only sensitive to the function's behavior at its singular points and at infinity. Especially for the nonrational case it is easy to dress a function which passes the test by something which does not alter this behavior.

The singularity confinement test is definitely a useful tool for identifying potentially integrable systems. It is probably necessary but, in the light of the present results, it appears to be insufficient. Of course, for a given map, the situation would be settled if one could establish any of the constructive properties associated with integrability, such as Lax pair, superposition principle, conservation laws, but in practice this is very difficult.

It is therefore important to continue developing and refining methods for algorithmic testing of integrability. For rational maps one such refinement is presented in this letter: one should look at the growth of the degree of the map (when written in the projective space): if the degree grows faster than polynomially (non vanishing "algebraic entropy"), it is likely that the system is not integrable.

Part of this work was done when J.H. was visiting the Institute of Computational Mathematics and Scientific Engineering Computing in Beijing. J.H. would like to thank M. Ablowitz, B. Grammaticos, X.B. Hu, S.H. Wang and Y.X. Yuan for discussions.

[1] M. Henon Numerical study of quadratic area preserving maps Q. J. Appl. Math. 27 291-312 (1969). Chaotic behaviour of deterministic systems Eds. G Iooss, R.H.G Helleman and R. Stora, Les Houches session XXXVI, North Holland 1983.

[2] A. Nagai and J. Satsuma, Discrete soliton equations and convergence acceleration algorithms, Phys. Lett. A 209, 305-312 (1995).

[3] S.V. Kovalevska, Sur le problème de la rotation d'un corps solide autour d'un point fixe, Acta Math. 12, (1889), pp 177-232.

[4] P. Painlevé Sur les équations différentielles d'ordre quelconque à points critiques fixes, C. R. Acad. Sc. Paris, 130 (1900), pp 1112-1115.

[5] A. Ramani, B. Grammaticos, and A. Bountis, The Painlevé property and singularity analysis of integrable and non-integrable systems, Phys. Rep. 180, 159-245 (1989).

[6] R. Conte The Painlevé approach to nonlinear ordinary differential equations, in "The Painlevé property, one century later", CRM Series in Mathematical Physics, Springer, Berlin (1998), and solv-int/9710020.

[7] B. Grammaticos, A. Ramani, and V. Papageorgiou, Do Integrable Mappings Have the Painlevé Property?, Phys. Rev. Lett. 67, 1825-1828 (1991).

[8] A. Ramani, B. Grammaticos and J. Hietarinta, Discrete Versions of the Painlevé Equation, Phys. Rev. Lett. 67, 1829-1831 (1991). B. Grammaticos, F.W. Nijhoff, and A. Ramani, Discrete Painlevé Equations, in "The Painlevé property, one century later", CRM Series in Mathematical Physics, Springer, Berlin (1998).

[9] M. Bellon, C.-M. Viallet, in preparation.

[10] G. Falqui, C.-M. Viallet, Singularity, complexity, and quasi-integrability of rational mappings, Com. Math. Phys. 154 111-125 (1993).

[11] V.I. Arnold, Dynamics of complexity of intersections, Bol. Soc. Bras. Mat. 21 1-10 (1990).

[12] A.P. Veselov, Growth and Integrability in the Dynamics of Mappings, Com. Math. Phys. 145 181-193 (1992).

[13] S. Boukraa, J-M. Maillard, and G. Rollet, Integrable mappings and polynomial growth, Physica A 209 162222 (1994).

[14] A.S. Fokas, B. Grammaticos, and A. Ramani, From Continuous to Discrete Painlevé Equations, J. Math. Anal. Appl. 180, 342-360 (1993). 